\title{
In Vitro Wheat Immature Spike Culture Screening Identified Fusarium Head Blight Resistance in Wheat Spike Cultured Derived Variants and in the Progeny of Their Crosses with an Elite Cultivar
}

\author{
Chen Huang, Manu P. Gangola, H. Randy Kutcher, Pierre Hucl, Seedhabadee Ganeshan, and \\ Ravindra N. Chibbar (D)* \\ Department of Plant Sciences, University of Saskatchewan, 51 Campus Drive, Saskatoon, S7N 5A8, Saskatchewan, \\ Canada
}

(Received on July 16, 2020; Revised on September 24, 2020; Accepted on October 20, 2020)

Fusarium head blight (FHB) is a devastating fungal disease of wheat (Triticum aestivum L.). The lack of genetic resources with stable FHB resistance combined with a reliable and rapid screening method to evaluate FHB resistance is a major limitation to the development of FHB resistant wheat germplasm. The present study utilized an immature wheat spike culture method to screen wheat spike culture derived variants (SCDV) for FHB resistance. Mycotoxin concentrations determined by liquid chromatography-tandem mass spectrometry (LC-MS/MS) correlated significantly $(P<0.01)$ with FHB severity and disease progression during in vitro spike culture. Selected SCDV lines assessed for FHB resistance in a Fusarium field disease nursery in Carman, Manitoba, Canada in 2016 showed significant $(P<0.01)$ correlation of disease severity to the in vitro spike culture screening method. Selected resistant SCDV lines were also crossed with an elite $\mathrm{cv}$. CDC Hughes and the progeny of $F_{2}$ and $B C_{1} F_{2}$ were screened by high resolution melt curve (HRM) analyses for the wheat UDPglucosyl transferase gene (TaUGT-3B) single nucleotide polymorphism to identify resistant (T-allele) and

\footnotetext{
*Corresponding author.

Phone) +1-306-966-4969, FAX) +1-306-966-5015

E-mail) ravi.chibbar@usask.ca

ORCID

Ravindra N. Chibbar

https://orcid.org/0000-0002-2282-5236

(c) This is an Open Access article distributed under the terms of the Creative Commons Attribution Non-Commercial License (http:// creativecommons.org/licenses/by-nc/4.0) which permits unrestricted noncommercial use, distribution, and reproduction in any medium, provided the original work is properly cited.
}

Articles can be freely viewed online at www.ppjonline.org. susceptible (G-allele) markers. The progeny from the crosses were also screened for FHB severity using the immature spike culture method and identified resistant progeny grouped according to the HRM genotyping data. The results demonstrate a reliable approach using the immature spike culture to screen for FHB resistance in progeny of crosses in early stage of breeding programs.

Keywords : 3-acetyldeoxynivalenol, deoxynivalenol, deoxynivalenol-3-glucoside, Fusarium head blight, wheat

Handling Editor : Cheol Seong Jang

Fusarium head blight (FHB) or scab is a major disease of wheat (Triticum aestivum L.) and is predominantly caused by Fusarium graminearum Schwabe [telomorph: Gibberella zeae (Schw.) Petch] (Schroeder and Christensen, 1963). Although other Fusarium spp., including $F$. avenaceum, $F$. culmorum, and $F$. poae, also cause FHB, they are less frequently reported, and the disease incidence and severity of FHB they cause is lower than that of $F$. graminearum (Parry et al., 1995). Immature wheat spikes are conducive to infection by Fusarium spp. at the flowering stage as a result of rainfall and high humidity, warm temperatures, and the presence of Fusarium infected crop residues. Within five to six hours after landing on the flowering wheat spikes the macroconidia begin to germinate on wheat glumes, while airborne spores enter plant tissue through the base of palea and lemma, natural openings, or degenerating anthers to initiate infection (Bushnell et al., 2003). The disease affects grain development resulting in light shriveled kernels with reduced yield (McMullen et al., 1997), which in small 
grain cereals can be up to $74 \%$ (McMullen et al., 1997, 2012; Parry et al., 1995).

Fusarium infection of wheat also leads to accumulation of the trichothecene group of mycotoxins such as deoxynivalenol (DON), 3-acetyldeoxynivalenol (3-ADON), 15-acetyldeoxynivalenol (15-ADON), and nivalenol (Huang et al., 2019; Kurata and Ueno, 1984; Marasas et al., 1984). Based on the different types of major mycotoxins produced, Fusarium graminearum strains have been recognized as chemotypes (reviewed in Desjardins, 2006). Among these chemotypes, the prevalence of 3-ADON chemotypes has been observed to be increasing from east to west in Canada (Ward et al., 2008; Xue et al., 2004). Thus, besides monitoring the dynamics of the chemotype populations, it is imperative to continue development of new resistant wheat varieties to keep pace with the changing Fusarium graminearum population. In addition to the confounding nature of the chemotypes, FHB resistance has been variously categorized, with five types currently recognized (Boutigny et al., 2008; Mesterházy et al., 1999). Types I and II resistance were initially proposed to refer to resistance against initial infection and FHB spread, respectively (Schroeder and Christensen, 1963). Subsequently, three other types have been recognized wherein ability to resist to kernel infection (Type III), tolerance to infection (Type IV), and resistance to DON accumulation (Type V) (Mesterházy, 2002; Miller et al., 1985).

Irrespective of the types of FHB resistance recognized, the challenge has generally been evaluation of FHB resistance in breeding programs, especially in field trials. While genotypic selection methods based on molecular markers have facilitated selection of resistant lines (Steiner et al., 2017), phenotypic evaluations at the whole plant level under field conditions are still needed to confirm genotypic selections. However, such whole plant level evaluations are often influenced by inconsistent disease pressure from year to year due to unpredictable environments and have led to the development of a number of other potential inoculation and screening methods for FHB. The most accepted inoculation method to date is the corn spawn strategy (for review see Imathiu et al., 2014). Some of the screening methods have relied on in vitro assays due to their simplicity and continue to be explored as alternatives for FHB screening. Detached leaf assays (Diamond and Cooke, 1999), seedling assays (Mesterházy, 1987), DON-based responses (Buerstmayr et al., 1997) and more recently immature spike culture assays (Sharma et al., 2018) are some of the in vitro methods used to assess FHB resistance.

Notwithstanding the screening methods employed, it is imperative to provide adequate validation for the selected method and demonstrate its consistency over generations and under variable field conditions. The ultimate goal of the FHB disease resistance development strategy is the utilization of genetic improvement and agronomic management practices to maximize crop performance under FHB disease pressure (Beres et al., 2018). Among the genetic strategies that can be undertaken are the generation and identification of new genetic resources for FHB resistance. We recently developed an ethyl methane sulfonate treated spike culture derived variant (SCDV) wheat population (Ganeshan and Chibbar, 2017) based on an immature wheat spike culture method to study grain development (Ganeshan et al., 2010). The immature spike culture method was further adapted to evaluate FHB severity by point inoculation of the spikelets of the SCDV lines (Sharma et al., 2018). The in vitro spike culture method is simple, rapid, environment-independent, and is less resource demanding in terms of time and labour compared to field-based screening (Huang et al., 2020). To further support the validity of the immature spike culture screening method for FHB disease severity assessment and resistance identification, the objectives of this study were to (1) assess FHB severity in $\mathrm{M}_{4} \mathrm{SCDV}$ lines under field conditions in one location in Carman, Manitoba, Canada in 2016 and in parallel using immature spike cultures of the corresponding $\mathrm{M}_{4}$ lines for comparison; (2) assess FHB severity in immature spike cultures of progeny derived from crosses of selected SCDV lines with elite cv. PT588 (CDC Hughes); and (3) use high resolution melt curve (HRM) to analyze occurrence of the single nucleotide polymorphic (SNP) marker in the UDP-glucosyl transferase (TaUGT-3B) gene, shown to be associated with FHB resistance (Sharma et al., 2018) in progeny of selected crosses. UDP-glucosyl transferase isolated from Arabidopsis thaliana, was shown to detoxify DON (Poppenberger et al., 2003). In wheat, it was hypothesized that the FHB resistance QTL, Qfhs.ndsu$3 B S$, encoded or regulated this enzyme (Lemmens et al., 2005). Subsequently, a gene (TaUGT3) was cloned and characterized in the wheat variety Wangshuibai, shown to detoxify DON and located on group 3 chromosomes of wheat (Lulin et al., 2009). Based on the latter study and our previous study (Sharma et al., 2018) we therefore decided to focus on the TaUGT-3B gene.

\section{Materials and Methods}

Plant materials and point inoculation of spikelets. A previously developed population of ethyl methane sulfonate mutagenized SCDV (Ganeshan and Chibbar, 2017) was used in this study. Based on consistent performance among replicates of $M_{3}$ generation of 134 SCDV lines in 
another study (Sharma et al., 2018), 53 were randomly selected based on a range of resistance to susceptibility reactions to FHB and also to have a manageable number of lines for in vitro inoculation for the present study. Sumai-3 and AC Nanda were used as FHB resistant and susceptible controls, respectively. The optimized in vitro spike culturebased technique as previously described (Ganeshan and Chibbar, 2017; Ganeshan et al., 2010, 2012) was used for FHB disease severity (Sharma et al., 2018). Briefly, $\mathrm{M}_{3}$ seeds from each of the selected 53 SCDV lines were grown in the greenhouse (University of Saskatchewan, Saskatoon, SK, Canada) during May to July 2015. At the onset of heading at least 20 immature spikes were collected for each of the SCDV lines, grown on spike culture media, and point-inoculated with a moderately virulent 3-ADON chemotype (M7-07-1) of $F$. graminearum (Huang et al., 2019). The remaining non-inoculated spikes were allowed to grow to maturity to obtain the $\mathrm{M}_{4}$ seeds. From the 53 $\mathrm{M}_{3}$ SCDV lines $\mathrm{M}_{4}$ seeds from 13 (11 FHB resistant and 2 susceptible) were selected based on their contrasting FHB resistance for in vitro spike culture and field-based experiments.

FHB disease severity was recorded at 5, 7, 9, and 11 days after inoculation (DAI). Point inoculated spikelets (from at least three spikes) of several lines $(80,93,123,136, \mathrm{AC}$ Nanda, 202, 250, 252, 256, and Sumai-3) were collected at 5, 7, 9 and 11 DAI, quick frozen in liquid nitrogen and stored at $-80^{\circ} \mathrm{C}$ for use in the determination of accumulation of mycotoxins. The selected lines included four susceptible $(80,93,123$, and 136) and four resistant lines (202, 250, 252, and 256) SCDV lines. Since these lines were previously studied (Sharma et al., 2018), only these eight representative lines were used along with the respective controls lines and also to keep the number of lines tested manageable. The leaves from plants of these SCDV lines at the three-leaf stage were also collected, washed with ethanol $(80 \% \mathrm{v} / \mathrm{v})$ and stored at $-80^{\circ} \mathrm{C}$ for subsequent DNA extraction.

FHB screening of in vitro spike cultures from $\mathrm{M}_{4} \mathrm{SCDV}$ lines. For the spike culture experiment, selected resistant $(66,81,91,98,100,135,192,202,224,245$, and 250) and susceptible (124 and 235) $\mathrm{M}_{4}$ lines were grown in the greenhouse (University of Saskatchewan, Saskatoon, SK, Canada) under a $16 / 8 \mathrm{~h}$ day/night cycle with average daily temperature of $27.3 / 20.6^{\circ} \mathrm{C}$ and relative humidity of $50.1 / 70.5 \%$ during May to July 2016. AC Nanda (susceptible) and Sumai-3 (resistant) were used as controls. Immature spikes were collected at Feekes' growth stage 10.5 (Feekes, 1941), processed and FHB severity recorded at
$5,7,9$, and 11 DAI as previously described (Sharma et al., 2018).

Field evaluation of $\mathbf{M}_{4} \mathrm{SCDV}$ plants. A field experiment with the selected $\mathrm{M}_{4} \mathrm{SCDV}$ lines was performed during the May to August growing period in 2016 in an FHB field nursery at Carman, Manitoba, Canada $\left(49^{\circ} 30^{\prime} \mathrm{N}, 98^{\circ} 0^{\prime} \mathrm{W}\right.$, elevation $262 \mathrm{~m}$ ). The average daily maximum/minimum temperature in Carman was $23.1 / 10.3^{\circ} \mathrm{C}$, with total precipitation of $282.2 \mathrm{~mm}$ during the growing period. The Carman FHB Nursery is located in the Red River Valley that naturally has very high humidity. However, the field also had overhead irrigation to provide adequate humidity when needed to facilitate FHB infection and spread. The field trial was performed with two replications in a randomized complete block design as previously described (Ruan et al., 2012). The plants were inoculated by spreading corn spawn (Gilbert and Woods, 2006) ( $\sim 4$ g per hill) infected with 3-ADON and 15-ADON chemotypes 10 days prior to anthesis of the plants. FHB incidence and severity were recorded to calculate a Visual Rating Index (VRI) as, VRI $=(\%$ incidence $\times \%$ severity $) / 100$, as described (Stack and McMullen, 1995) 15 days after inoculation.

Generation of $\mathrm{BC}_{1} \mathrm{~F}_{2}$ progeny from crosses of SCDV lines with elite cv. CDC Hughes (PT588) and assessment of FHB resistance using immature spike culture. Four FHB resistant SCDV lines (202, 244, 250 and 256) based on in vitro spike culture inoculation experiments were crossed with CDC Hughes (Canadian Food Inspection Agency certificate \#5952), an elite Canada Western Red Spring wheat variety with moderate resistance to FHB. The $\mathrm{F}_{2}$ as well as the $\mathrm{BC}_{1} \mathrm{~F}_{2}$ progeny was analyzed for the TaUGT-3B SNP and FHB reaction by immature spike culture. A total of 191 wheat plants were obtained from eight cross combinations, with varying number of plants derived from each combination. In the first four crosses, the four selected resistant SCDV wheat lines were the female parents, while the common wheat cultivar CDC Hughes was the male parent. In the last four crosses $\left(\mathrm{BC}_{1}\right)$, the female parent was $\mathrm{CDC}$ Hughes, while $\mathrm{F}_{1}$ plants were the male parents.

Three spikes were cut at heading stage from each of 191 wheat plants. After four days of culture in spike culture media (Ganeshan et al., 2010, 2012), the wheat spikes were inoculated with Fusarium graminearum 3-ADON strain M7-07-1 as described (Huang et al., 2019). Briefly, after inoculation, the plants were covered with clear plastic bags and placed in a Sanyo MIR-351H incubator (Sanyo Canada, Inc., Concord, ON, Canada) at 16-h day and 8-h night 
cycle, temperatures $24^{\circ} \mathrm{C}$ (night) and $26^{\circ} \mathrm{C}$ (day). After 2.5 days of incubation, the bags were removed, and at $8 \mathrm{DAI}$, disease severity assessed. In our previous studies (Huang et al., 2019, 2020) we determined that disease severity at 9 DAI was more informative and was therefore used for disease severity recording.

DNA extraction and HRM analysis for the TaUGT$3 B$ SNP. Leaf samples, stored at $-80^{\circ} \mathrm{C}$, were used for DNA extraction using a commercial kit (Qiagen DNeasy Plant Mini Kit, Qiagen, Germantown, MD, USA) following the manufacturer's protocol. The DNA isolated was used for HRM analysis as previously described (Sharma et al., 2018). Briefly, a pair of primers (TaUGT-3BF1 and TaUGT-3BR2) was used to amplify a fragment of 1,882 bp encompassing the TaUGT-3B SNP location at 1,558 bp from the start codon. The PCR fragment amplified was used in a nested PCR for HRM analysis of the SNP using primers, TaUGT-3BHF1 and TaUGT-3BHR1. Sumai-3 and AC Nanda were used as controls in all the HRM analyses.

The reaction volume $(20 \mu \mathrm{l})$ for HRM contained $10 \mu \mathrm{l}$ of HRM super mix from Precision Melt Supermix (BioRad laboratories, Inc., Hercules, CA, USA), $10 \mu \mathrm{M}$ of each pair of primers, $1.0 \mu \mathrm{l}$ PCR products from the first round regular PCR as DNA template and sterile deionized water. The HRM analysis was done in two consecutive steps in a CFX96 Real-Time System (Bio-Rad Laboratories (Canada) Ltd., Mississauga, ON, Canada). The two steps included the amplification and melting curve assays. The amplification step consisted of a $10 \mathrm{~min}$ initial denaturation, followed by 40 cycles of $95^{\circ} \mathrm{C}$ for $15 \mathrm{~s}$ and $60^{\circ} \mathrm{C}$ for $30 \mathrm{~s}$. The melt step consisted of denaturation for $10 \mathrm{~s}$ at $95^{\circ} \mathrm{C}$ followed by annealing for $1 \mathrm{~min}$ at $60^{\circ} \mathrm{C}$, and melting stage in increments of $0.1^{\circ} \mathrm{C}$ from $60^{\circ} \mathrm{C}$ to $95^{\circ} \mathrm{C}$, followed by the final annealing step for $1 \mathrm{~min}$ at $60^{\circ} \mathrm{C}$. The melt data were analyzed using the Bio-Rad Precision Melt Analysis software (Bio-Rad Laboratories (Canada) Ltd., Mississauga, $\mathrm{ON}$, Canada) for generation of high-resolution melting curves.

Statistical analysis. Multiple comparisons using Tukey's test were performed to compare disease severity between resistant and susceptible genotypes at multiple DAI. Correlation analysis was performed to analyze the association among various traits studied such as the severity, incidence and visual rating index, whereas multivariate analysis was performed to group the genotypes based on the parameters studied. The statistical analyses were performed using Proc Mixed of software SAS version 9.4 (SAS Institute, Cary, NC, USA) and Minitab 16 statistical software (Minitab Inc., State College, PA, USA) for correlation analyses.

\section{Results}

FHB severity in $53 \mathrm{M}_{3} \mathrm{SCDV}$ lines and mycotoxins accumulation in selected resistant and susceptible lines. The $53 \mathrm{M}_{3} \mathrm{SCDV}$ lines evaluated using the in vitro spike culture technique varied for FHB resistance $(P<0.001)$ from $24.3 \%$ to $75.5 \%, 29.3 \%$ to $80.1 \%, 43.7 \%$ to $84.1 \%$, and $56.7 \%$ to $100 \%$ with averages of $43.8 \%, 60.5 \%, 71.7 \%$ and $89.4 \%$ at $5,7,9$, and 11 DAI, respectively (Fig. 1,

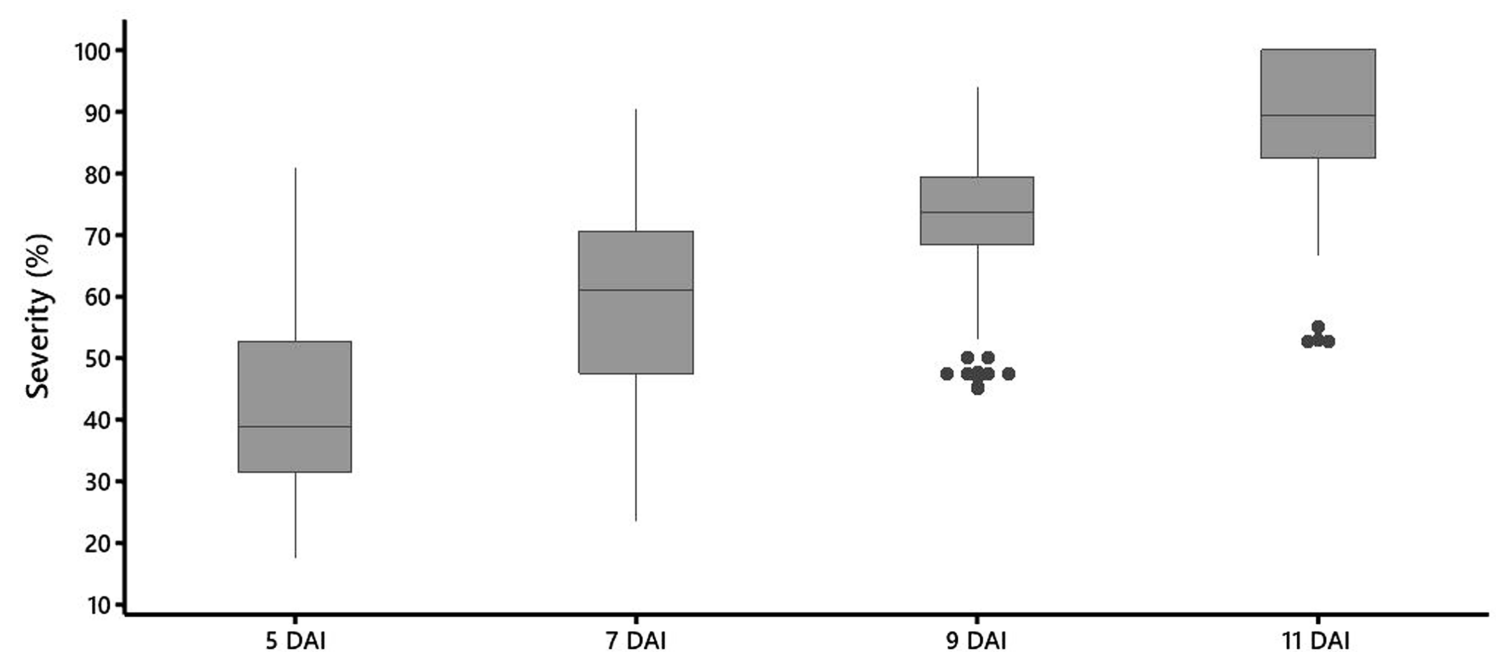

Fig. 1. Box-plot analysis of $53 \mathrm{M}_{3}$ spike culture derived variants showing variation for Fusarium head blight severity at 5, 7, 9, and 11 days after inoculation (DAI). The dark circles represent the outliers, calculated as the data points out of 1.5 times the interquartile range. 

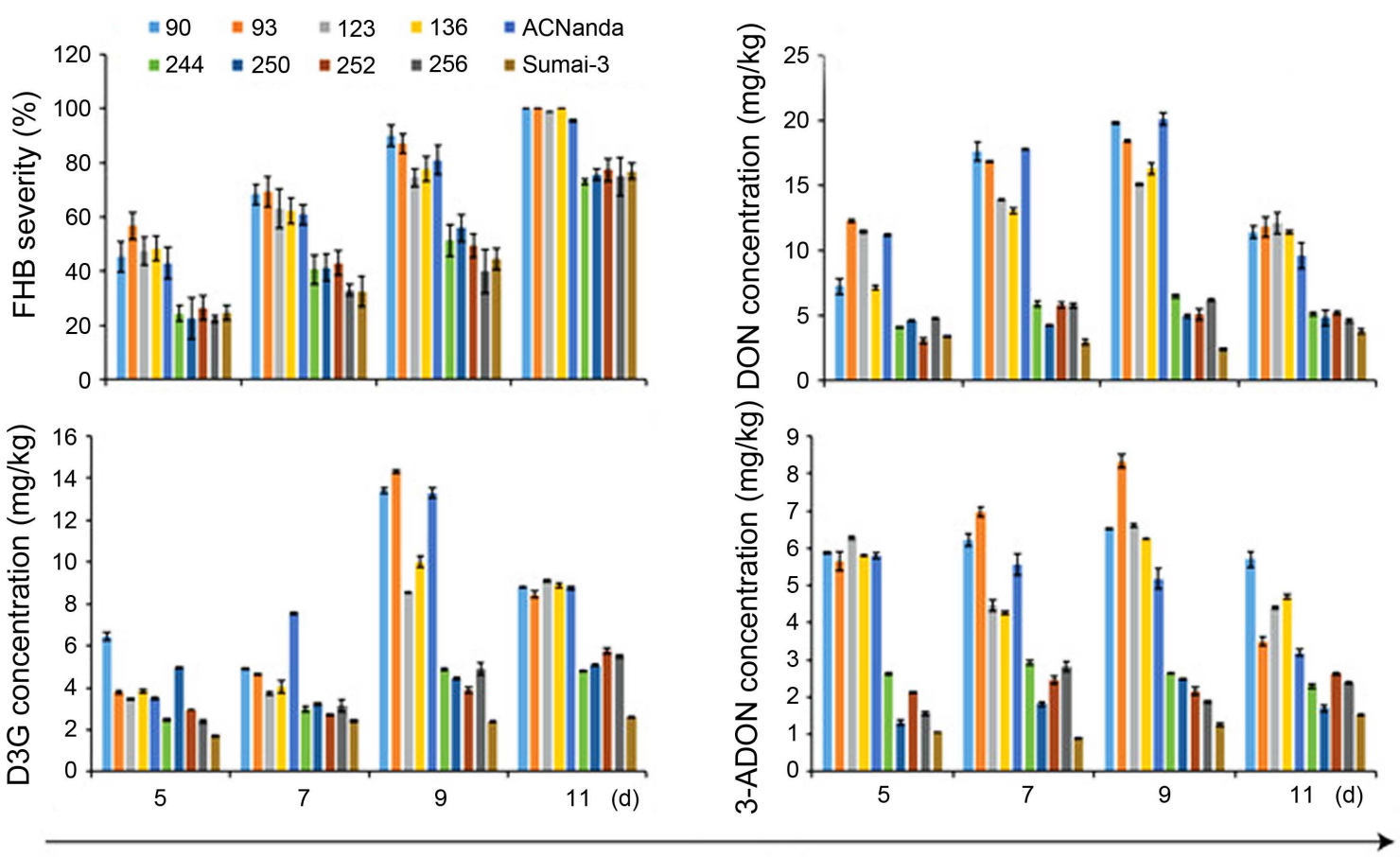

Fig. 2. Bar-graphs showing Fusarium head blight (FHB) severity (\%), and concentrations ( $\mathrm{mg} / \mathrm{kg}$ ) of deoxynivalenol (DON), deoxynivalenol-3-glucoside (D3G), and 3-acetyldeoxynivalenol (3-ADON) in FHB resistant and susceptible $\mathrm{M}_{3}$ spike culture derived variants lines at 5, 7, 9, and 11 days after inoculation.

Supplementary Table 1). The replication effect was not significant at any stage after inoculation; however, the most pertinent differences $(P \leq 0.001)$ among the SCDV lines were observed at 7 and 9 DAI.

Based on FHB severity among the 53 SCDV lines in in vitro spike culture inoculation experiments, four resistant and four susceptible lines were selected for mycotoxin analyses (Fig. 2). The FHB severity ranged from $22.4 \%$ to $26.4 \%, 32.5 \%$ to $43.0 \%, 40.0 \%$ to $56.1 \%$, and $73.0 \%$ to $77.5 \%$ in the FHB resistant SCDV lines selected at 5, 7, 9 and 11 DAI, respectively. In the susceptible lines, FHB severity ranged from $43.0 \%$ to $56.9 \%, 61.0 \%$ to $69.4 \%$, $74.6 \%$ to $80.1 \%$, and $95.6 \%$ to $100.0 \%$ at $5,7,9$ and 11 DAI, respectively. Among the accumulated mycotoxins in the grain, DON accumulation was predominant, followed by $3-A D O N$ in resistant as well as susceptible lines (Fig. 2). The 15-ADON and nivalenol mycotoxins were either absent or not detected. The glucosylated product of DON, deoxynivalenol-3-glucoside (D3G), was also detected. Comparing the resistant and susceptible lines, the latter accumulated higher concentrations of DON and 3-ADON ( $P$ $\leq 0.001)$ at $5,7,9$, and 11 DAI. Accumulation of D3G differed between the resistant and susceptible lines $(P \leq 0.001)$ only at 7,9 , and 11 DAI.
FHB disease severity in $\mathbf{M}_{4}$ SCDV lines in field and in vitro culture experiments. A field experiment with selected resistant and susceptible $\mathrm{M}_{4}$ SCDV lines was conducted in Carman, Manitoba, Canada in 2016 for preliminary comparison of in vitro culture method and field assessment method, with expected detailed multi-year and multi-location trials in the future for confirmation. The occurrence of high disease incidence led to significant FHB severity delineation between the resistant and susceptible genotypes $(P<0.05)$, with FHB severity ranging from 10 to $55 \%$ and VRI from 4.0 to $49.5 \%(P<0.01)$ (Fig. 3A).

The severity data from the field trial when compared to FHB severity data from the in vitro spike culture method of evaluation was in agreement in its ability to discern resistant and susceptible genotypes (Fig. 3B). The severity data showed high correlation $(P<0.05)$ with the in vitro data for all four DAI (Table 1).

SNP marker identification in progeny of resistant SCDV lines $\times$ CDC Hughes crosses and frequencies. A total of 191 wheat plants from eight cross combinations, with varying number of plants derived from each combination, were analyzed for SNP variation in TaUGT-3B gene by real-time PCR and HRM, wherein the AC Nanda SNP 

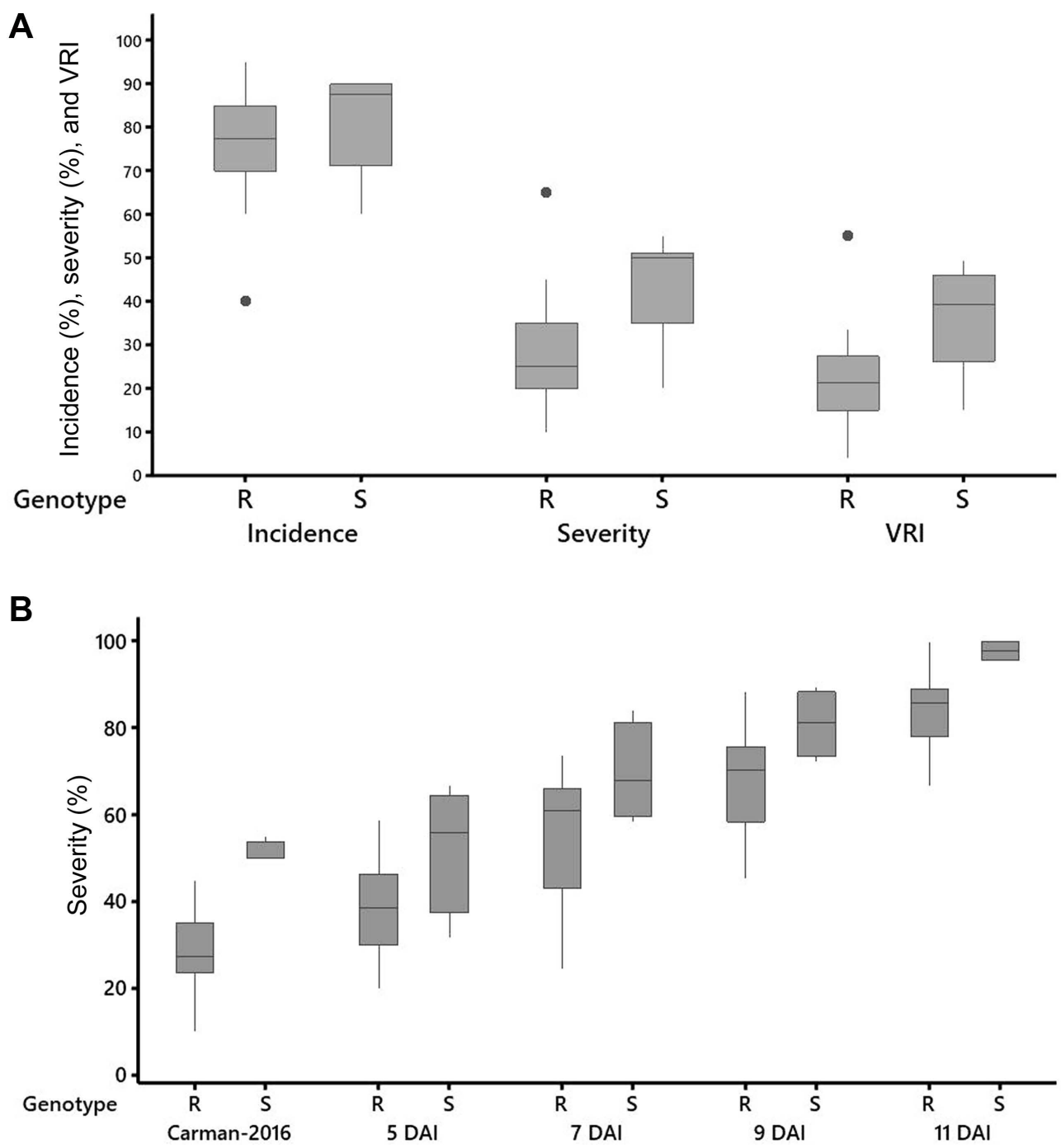

Fig. 3. Box-plot analysis Fusarium head blight (FHB) disease data in field trial in Carman, Manitoba, Canada in 2016 and immature spike culture screening. (A) FHB incidence, severity and visual rating index (VRI) in field trial in 2016. (B) Comparison of severity data from Carman field trial and immature spike culture screening method. DAI, days after inoculation; R, resistant; S, susceptible.

(nucleotide G) was considered the wild type and the alternative SNP (nucleotide T) was the mutation corresponding to the Sumai-3 TaUGT-3B gene (Sharma et al., 2018). In the first four crosses, the four selected resistant SCDV wheat lines $(202,244,250$ and 256) were used as the female parents and the elite wheat cultivar CDC Hughes as the male parent. In the other four crosses $\left(\mathrm{BC}_{1}\right)$, the female parent was $\mathrm{CDC}$ Hughes, while $\mathrm{F}_{1}$ plants were the male parents. The progeny from the $\mathrm{BC}_{1} \mathrm{~F}_{2}$ showed SNP variation, which distinguished between the homozygotes and heterozygotes (Fig. 4).

Among the $F_{2}$ progeny of the four crosses, the highest

Table 1. Correlation analyses of data from field trial and the immature spike culture screening method for evaluation of FHB severity

\begin{tabular}{lclllll}
\hline & SEV-C-2016 & INC-C-2016 & VRI-C-2016 & SEV-5 DAI & SEV-7 DAI & SEV-9 DAI \\
\hline INC-C-2016 & $0.527^{* *}$ & & & & & \\
VRI-C-2016 & $0.850^{* * *}$ & $0.668^{* * *}$ & & & & \\
SEV-5 DAI & $0.438^{*}$ & $0.260 \mathrm{~ns}$ & $0.381 \mathrm{~ns}$ & & & \\
SEV-7 DAI & $0.504^{*}$ & $0.321 \mathrm{~ns}$ & $0.551^{* *}$ & $0.738^{* * *}$ & & \\
SEV-9 DAI & $0.437^{*}$ & $0.254 \mathrm{~ns}$ & $0.501^{*}$ & $0.562^{* *}$ & $0.850^{* * *}$ & \\
SEV-11 DAI & $0.606^{* *}$ & $0.415^{*}$ & $0.567^{* *}$ & $0.513^{*}$ & $0.599^{* *}$ & $0.486^{*}$ \\
\hline
\end{tabular}

*Significant at $P<0.05, * *$ Significant at $P<0.01, * * *$ Significant at $P<0.001$.

FHB, Fusarium head blight; SEV, severity; INC, incidence; VRI, visual rating index; DAI, days after inoculation; ns, not significant. 


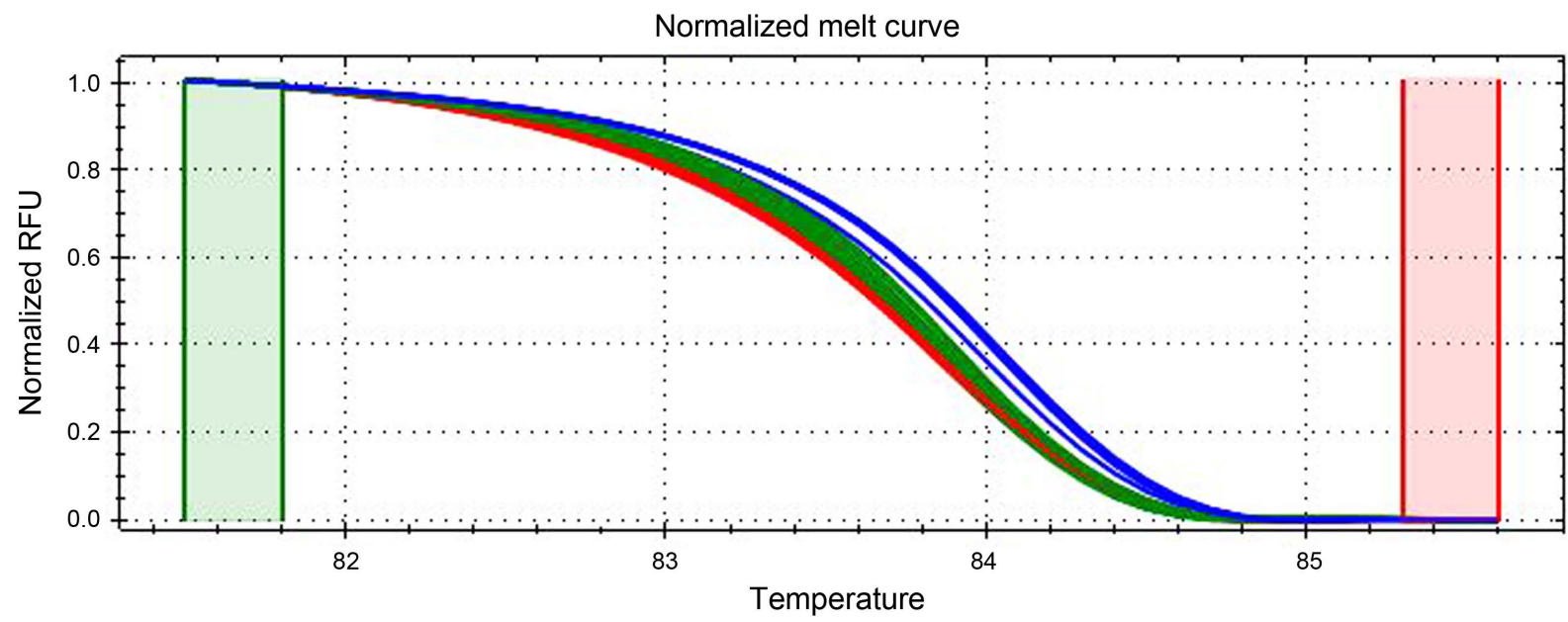

Fig. 4. Representative high resolution melt curve (analysis for the TaUGT-3B single nucleotide polymorphism (SNP) from progeny of different cross combinations between resistant wheat spike culture derived variants lines and the Fusarium head blight susceptible elite wheat cv. CDC Hughes. Blue curves, green curves, and red curves indicate homozygous lines carrying the AC Nanda SNP; homozygous mutant lines carrying the Sumai-3 SNP; and heterozygous lines. RFU, relative fluorescence unit.

frequency of the homozygous mutation SNP was detected in the FHB250/PT588 cross, with nine homozygotes out of 26 plants tested, corresponding to $34.6 \%$ (Table 2). The lowest frequency of homozygotes was detected in FHB202/PT588, with only one homozygote, accounting for $5.9 \%$ of the $\mathrm{F}_{2}$ population. In FHB244/PT588 and FHB256/PT588, both crosses had two homozygotes with the same SNP type as Sumai-3, accounting for $9.0 \%$ and $10.5 \%$, respectively, of the progeny. The highest frequency of heterozygotes was detected in progeny of the FHB256/ PT588 cross, with $57.9 \%$ heterozygotes, followed by FHB250/PT588 (46.2\%), FHB202/PT588 (23.5\%), and
FHB244/PT588 (22.7\%). The heterozygote frequencies in the progeny from the four $\mathrm{BC}_{1} \mathrm{~F}_{2}$ populations were generally lower than in the progeny from the $\mathrm{F}_{2}$ populations, with percentages of $17.9 \%$ (PT588*2/FHB244), $17.4 \%$ (PT588*2/FHB250), 13.6\% (PT588*2/FHB256), and $23.5 \%$ (PT588*2/FHB202). There were no homozygous mutant SNP identified in progeny from PT588*2/FHB202 and PT588*2/FHB244 crosses.

FHB severity assessment in HRM-based progeny grouping from the eight cross combinations. FHB severity was assessed based on the grouping of the progeny from

Table 2. Summary of percentages of wild-type, heterozygous, and homozygous progeny for SNP in TaUGT-3B gene from crosses between elite wheat cultivar PT588 and FHB-resistant SCDV genotypes by HRM analysis

\begin{tabular}{|c|c|c|c|c|c|}
\hline $\begin{array}{l}\text { No. of cross } \\
\text { combinations }\end{array}$ & $\begin{array}{l}\text { Total no. of } \\
\text { progeny plants }\end{array}$ & Generation & $\begin{array}{c}\text { Wild type progeny } \\
\text { plants (AC Nanda SNP) } \\
(\%)\end{array}$ & $\begin{array}{l}\text { Homozygous mutation - } \\
\text { no. of progeny plants }(\%) \\
\text { (Sumai-3 SNP) }\end{array}$ & $\begin{array}{c}\text { Heterozygotes } \\
\text { mutation - no. of } \\
\text { progeny plants }(\%)\end{array}$ \\
\hline FHB202/PT588 & 17 & $\mathrm{~F}_{2}$ & $12(70.6)$ & $1(5.9)$ & $4(23.5)$ \\
\hline FHB244/PT588 & 22 & $\mathrm{~F}_{2}$ & $15(68.2)$ & $2(9.1)$ & $5(22.7)$ \\
\hline FHB250/PT588 & 26 & $\mathrm{~F}_{2}$ & $5(19.2)$ & $9(34.6)$ & $12(46.2)$ \\
\hline FHB256/PT588 & 19 & $\mathrm{~F}_{2}$ & $6(31.6)$ & $2(10.5)$ & $11(57.9)$ \\
\hline Total & 84 & $\mathrm{~F}_{2}$ & 38 & 14 & 32 \\
\hline PT588*2/FHB202 & 34 & $\mathrm{BC}_{1} \mathrm{~F}_{2}$ & $26(76.5)$ & $0(0.0)$ & $8(23.5)$ \\
\hline PT588*2/FHB244 & 28 & $\mathrm{BC}_{1} \mathrm{~F}_{2}$ & $23(82.1)$ & $0(0.0)$ & $5(17.9)$ \\
\hline PT588*2/FHB250 & 23 & $\mathrm{BC}_{1} \mathrm{~F}_{2}$ & $15(65.2)$ & $4(17.4)$ & $4(17.4)$ \\
\hline PT588*2/FHB256 & 22 & $\mathrm{BC}_{1} \mathrm{~F}_{2}$ & $13(59.1)$ & $6(27.3)$ & $3(13.6)$ \\
\hline Total & 107 & $\mathrm{BC}_{1} \mathrm{~F}_{2}$ & 77 & 10 & 20 \\
\hline Combined total & 191 & & & & \\
\hline
\end{tabular}

SNP, single nucleotide polymorphism; FHB, Fusarium head blight; SCDV, spike culture derived variants; HRM, high resolution melt curve. 

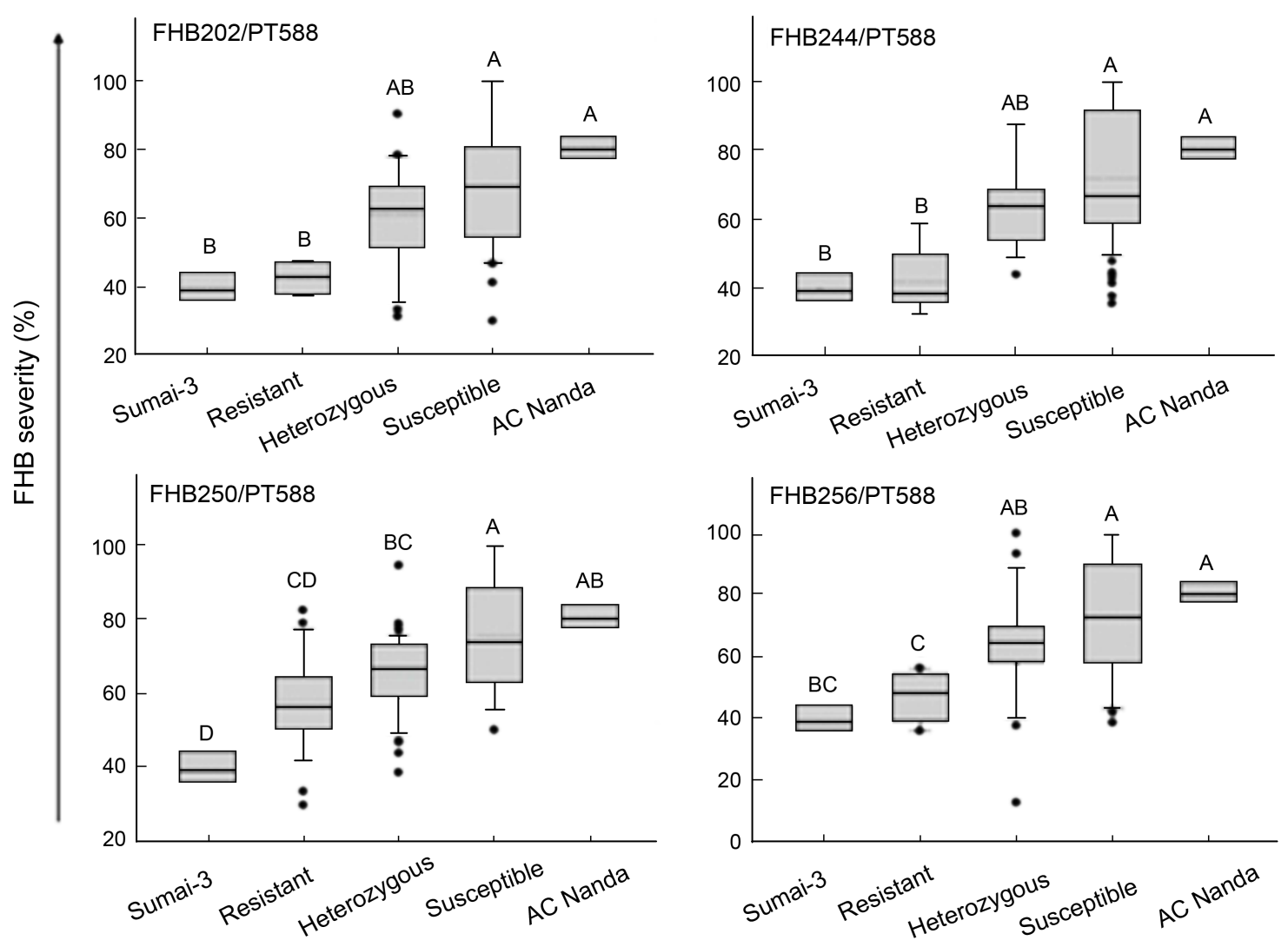

Fig. 5. The ANOVA results of Fusarium head blight (FHB) severity of different genotypic categories determined by high-resolution melt curve: Sumai-3, homozygous carriers (the mutant type of single nucleotide polymorphism [SNP], same as Sumai-3), heterozygotes genotypes, non-carriers (the wild type of SNP, same as AC Nanda) and AC Nanda at 9 days after inoculation of $F_{2}$ progeny from wheat population of different cross combinations between resistant wheat genotypes FHB202, FHB244, FHB250, FHB256 and the susceptible elite wheat cultivar PT588. Different letters indicate statistically significant differences of multiple levels of FHB severity $(P<0.01)$.

the crosses according to the occurrence of the TaUGT$3 B$ SNP in HRM. Progeny homozygous for T nucleotide SNP (similar to that in the TaUGT-3B gene of Sumai-3) was considered a carrier and those homozygous for the G nucleotide SNP (similar to that in the TaUGT-3B gene in AC Nanda) were grouped as non-carriers. In the cross FHB202/PT588, the disease severity of the resistant carrier genotypes was comparable to that of Sumai-3 (Fig. 5). Similarly, the non-carrier susceptible genotypes had disease severity similar to that of AC Nanda. The disease severity between carrier and non-carrier were significant $(P$ $<0.01)$, with a similar trend being in the carrier and noncarrier progeny of the FHB244/PT588 cross $(P<0.01)$, as revealed by ANOVA (Fig. 5). In the progeny of cross combinations FHB250/PT588 and FHB256/PT588, again there were no significant differences between Sumai-3 and carrier genotypes, as well as between non-carrier genotypes and AC Nanda, but there were differences $(P<0.01)$ between carrier and non-carrier genotypes.
In progeny of cross combinations PT588*2/FHB202 and PT588*2/FHB244, the homozygous carriers were not detected (Fig. 6). The heterozygous genotypes in both populations showed comparable FHB resistance to that of Sumai-3. There were differences between the non-carrier and heterozygous $(P<0.01)$ for both crosses. In progeny of the cross combinations PT588*2/FHB250 and PT588*2/ FHB256, the SNP carriers and Sumai-3 as well as noncarriers and AC Nanda did not show significant differences in disease severity; however, disease severity in carrier and non-carrier genotypes in both crosses differed $(P<0.01)$.

\section{Discussion}

A population of wheat SCDV developed previously (Ganeshan and Chibbar, 2017) was used for FHB resistance studies (Sharma et al., 2018) and further used in this study for FHB severity determination in field trials and in progeny of selected crosses using an immature spike culture screen- 

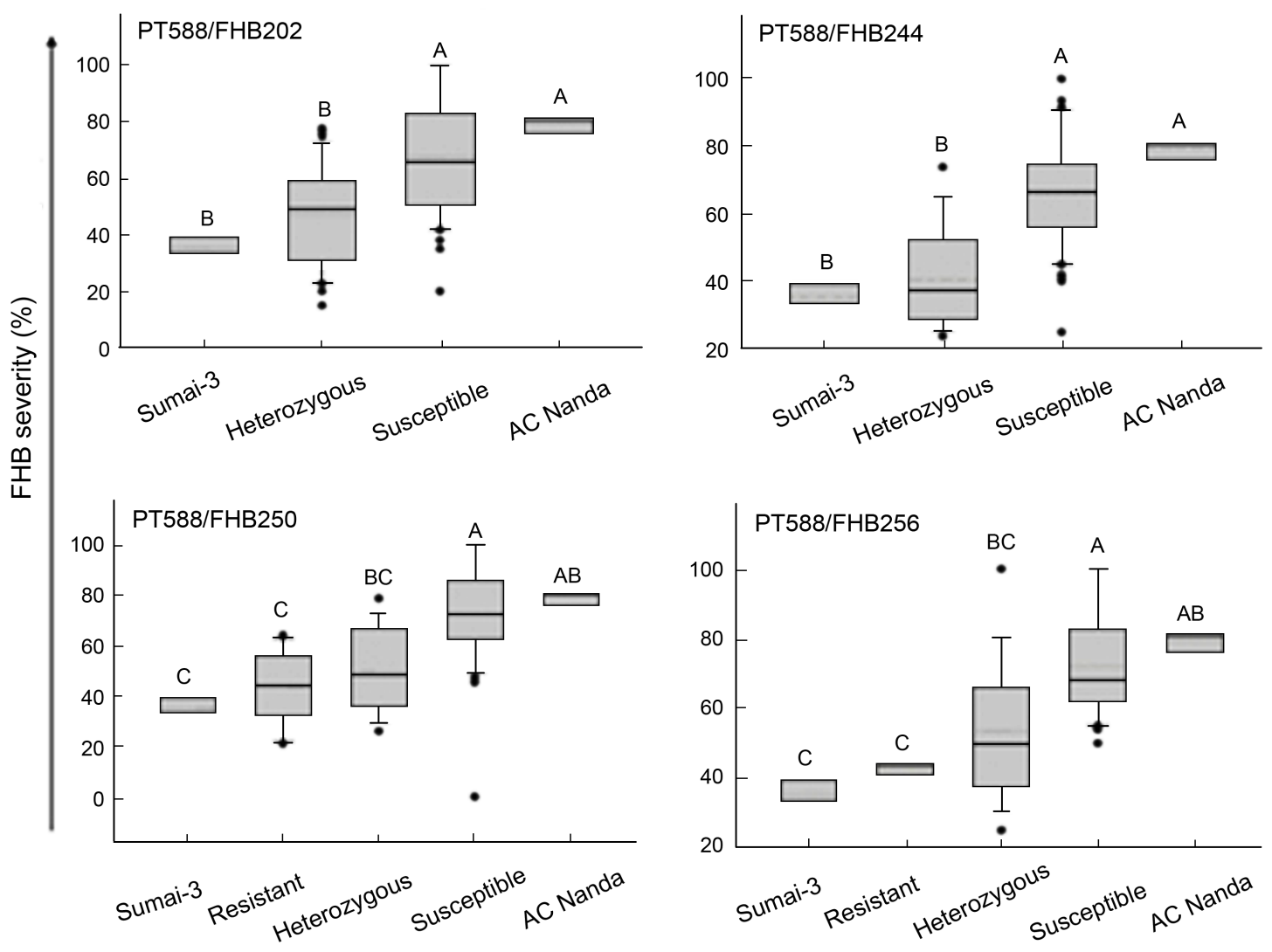

Fig. 6. The ANOVA results for Fusarium head blight (FHB) severity of different genotypic categories determined by high resolution melt curve: Sumai-3, homozygous carrier (the mutant type of single nucleotide polymorphism [SNP], same as Sumai-3), heterozygous genotypes, non-carrier (the wild type of SNP, same as AC Nanda) and AC Nanda at 9 days after inoculation (DAI) of BC $\mathrm{F}_{2}$ progenies from wheat population of different crossing combinations between FHB susceptible elite wheat cultivar PT588 and resistant wheat genotypes FHB202, FHB244, FHB250, FHB256. Different letters indicate statistically significant differences of multiple levels of FHB severity $(P<0.01)$.

ing method. The $53 \mathrm{M}_{3}$ generation SCDV lines used in this study were derived from $\mathrm{M}_{2}$ lines based on their FHB resistance (Sharma et al., 2018) and were confirmed in this study for their significant variation for FHB resistance. The FHB severity data correlated with those of the $\mathrm{M}_{2}$ SCDV lines. Lack of differences among replications at all days after point-inoculation of spikelets affirmed the repeatability of the spike culture technique in evaluating FHB resistance in vitro. The correlation between the $\mathrm{M}_{2}$ and $\mathrm{M}_{3}$ stages for FHB resistance or severity indicated a moderately to highly heritable trait that concurred with the heritability range of 0.5-0.9 for distinct FHB related parameters in wheat (Burt et al., 2015; Miedaner et al., 2017).

The selection of individuals from the progeny of a cross determines the success of a typical breeding program. Therefore, accurate and precise FHB evaluation is critical to identify resistant lines in the early stages of selection. At present, field-based inoculation (to the soil surface and into single florets) and other approaches including the detached leaf assay, the clip dipping method, the foliar spray method and pin-point inoculation have been used to evaluate FHB severity or resistance (Kumar et al., 2011; Shin et al., 2014; Yang et al., 1999). Field-based FHB screening is reliable, but it is affected by environmental conditions, and is time-consuming and labor-intensive. FHB mainly affects spikes at the flowering stage, therefore evaluating FHB resistance in detached leaves is an indirect approach and may affect the selection process. Consequently, an FHB screening method is required that combines the easy and fast approach of indirect methods with the reliability of field-based screening methods. An in vitro spike culture method was previously optimized (Ganeshan and Chibbar, 2017; Ganeshan et al., 2010, 2012), and subsequently demonstrated to be valuable for assessment of FHB severity (Sharma et al., 2018). The present study further confirmed the in vitro spike culture system for its accuracy and preci- 
sion by correlating FHB severity in the next generation $\mathrm{M}_{3}$ with mycotoxin accumulation by using selected FHB resistant lines (244, 250, 252, 256, and Sumai-3) and FHB susceptible lines (80, 93, 123, 136 and AC Nanda) at different DAI. The liquid chromatography-tandem mass spectrometry (LC-MS/MS) based approach for mycotoxin detection and quantification was optimized previously using immature wheat spike culture (Huang et al., 2020) and showed similar increased FHB disease development patterns corresponding to increased mycotoxin accumulation in FHB susceptible SCDV lines compared to the resistant lines. In agreement with the previous study (Huang et al., 2020) FHB severity and concentrations of DON, D3G and 3-ADON distinctly delineated FHB resistant and susceptible SCDV lines (Fig. 2) and further validated the immature spike culture screening method in the $\mathrm{M}_{3}$ generation. Also, as observed in our previous study (Huang et al., 2020), the decrease in mycotoxins at $11 \mathrm{DAI}$ compared to 5, 7, and 9 DAI is likely due to the senescing spikes prevent further growth of the Fusarium graminearum.

With the validation of the immature spike method of FHB evaluation over two generations from greenhouse grown plants, a field assessment was conducted in Carman, Manitoba, Canada in 2016 (Fig. 3A) to evaluate selected resistant and susceptible SCDV lines of the $\mathrm{M}_{4}$ generation in conventional FHB disease nursery. The corresponding selected $\mathrm{M}_{4}$ SCDV lines were also evaluated for FHB disease severity using the immature spike culture method from greenhouse grown plants (Fig. 3B). One of the major challenges to field assessment in disease nurseries is the generation of an adequate epidemic of FHB due to variable environmental conditions. As evident from the data (Fig. 3A), the trial in Carman, Manitoba in 2016 had good FHB disease incidence that translated into FHB severity and VRI capable of separating the resistant and susceptible lines. This could be attributed to a total precipitation of $282.2 \mathrm{~mm}$ with an average of $3.1 \mathrm{~mm} /$ day in Carman in 2016. High humidity or intensive precipitation events support the survival of the primary inoculum during the vegetative stage, and are conducive to infection during the anthesis stage of plant development, thus facilitating FHB disease development (reviewed in Audenaert et al., 2013; Beres et al., 2018; Shah et al., 2018). The FHB severity determinations using the in vitro spike culture technique was positively correlated with the field FHB disease severity assessments in Carman, in agreement with the conclusion of a previous study (Jin et al., 2013) wherein a strong positive correlation $(r=0.73, P<0.001)$ was observed between FHB severity in greenhouse and field experiments of 363 US winter wheat accessions. The strong correlation in Car- man in 2016 in this study supports the utilization of the in vitro spike culture method to evaluate FHB resistance or severity in the early stage selection for FHB resistance. However, further field trials in different locations would have to be conducted to further validate the results.

To demonstrate the effectiveness of the immature spike culture screening method to select FHB resistant germplasm, this study proceeded with advancing the resistant SCDV lines selected in a breeding experiment by crossing with the elite wheat cv. CDC Hughes (PT588). Combining a marker-assisted backcross approach, the introgression of the TaUGT-3B SNP in the backcross progeny was identified by HRM analysis (Fig. 4). The TaUGT-3B SNP was previously shown to be strongly associated with FHB resistance in SCDV lines (Sharma et al., 2018). The progeny with homozygous SNP T-allele (like in the FHB resistant Sumai-3 control genotype) showed reduced FHB disease severity $(P<0.01)$ compared to the progeny with homozygous SNP allele-G (like the FHB susceptible AC Nanda control genotype) in the $\mathrm{F}_{2}$ as well as $\mathrm{BC}_{1} \mathrm{~F}_{2}$ (Figs. 5, 6). In the $\mathrm{F}_{2}$ progeny depending on the cross, the presence of the $\mathrm{T}$ allele in homozygous genotypes led to FHB severity of $40-55 \%$ compared to $60-65 \%$ in heterozygous lines and about $70 \%$ in $\mathrm{G}$ allele carrying genotypes, indicating an important role for the " $\mathrm{T}$ " allele in conferring resistance. Similarly, in the $\mathrm{BC}_{1} \mathrm{~F}_{2}$ progeny depending on the cross, the homozygous $\mathrm{T}$ allele genotypes showed $40 \%$ FHB severity, while the heterozygous genotypes showed $45-50 \%$ and the $\mathrm{G}$ allele genotypes showed $65 \%$, again indicating a strong influence of the $\mathrm{T}$ allele. Furthermore, the $\mathrm{BC}_{1} \mathrm{~F}_{2}$ progeny carrying the homozygous $\mathrm{T}$ allele showed similar or lower FHB severity when the $\mathrm{F}_{1}$ SCDV lines were used as male and the PT588 as female compared to the progeny $\left(\mathrm{F}_{2}\right)$ when SCDV lines were used as female and CDC Hughes as male. The heterozygous progeny for the $\mathrm{T}$ allele also showed lower FHB severity in the $\mathrm{BC}_{1} \mathrm{~F}_{2}$ when the $F_{1}$ SCDV lines were used as male and the CDC Hughes as female. The source of variation for FHB resistance among SCDV lines was attributed to single nucleotide polymorphisms in two UDP-glucosyltransferase (UGT) genes, TaUGT-2B and TaUGT-3B (Sharma et al., 2018). The UDP-glucosyltransferase enzyme catalyzes the glycosylation of DON into DON-3-Glucoside (Lemmens et al., 2005), and was shown to be present by liquid chromatography in Fusarium infected samples (Berthiller et al., 2005).

In conclusion, the present study supports the in vitro spike culture technique to evaluate FHB disease severity and identify resistance. FHB resistant SCDV lines had reduced DON, 3-ADON, and D3G accumulation compared to that of FHB susceptible SCDV lines. The SCDV lines 
were also evaluated in a field experiment in Carman, Manitoba, Canada in 2016 and FHB incidence correlated with the immature spike culture severity data. The immature spike culture method has been shown in this study and our previous studies (Huang et al., 2020; Sharma et al., 2018) to be a simple, efficient and expedited alternative for early stage selection for FHB resistance in germplasm collections and breeding programs.

\section{Conflicts of Interest}

No potential conflict of interest relevant to this article was reported.

\section{Acknowledgments}

We are grateful to the former Cereal Research Centre, Agriculture and Agri-Food Canada, Winnipeg for providing the $F$. graminearum isolates. We gratefully acknowledge Professor Anita Brule-Babel, University of Manitoba, Winnipeg for help with field experiments in Carman, Manitoba, Canada. Financial support from Canada Research Chairs program and Natural Sciences and Engineering Research Council (NSERC) cooperative research project with Saskatchewan Wheat Development Commission (Sask Wheat) is gratefully acknowledged. The China Scholarship Council is greatly appreciated for the scholarship provided to the first author $(\mathrm{CH})$.

\section{Electronic Supplementary Material}

Supplementary materials are available at The Plant Pathology Journal website (http://www.ppjonline.org/).

\section{References}

Audenaert, K., Vanheule, A., Höfte, M. and Haesaert, G. 2013. Deoxynivalenol: a major player in the multifaceted response of Fusarium to its environment. Toxins (Basel) 6:1-19.

Beres, B. L., Brûlé-Babel, A. L., Ye, Z., Graf, R. J., Turkington, T. K., Harding, M. W., Kutcher, H. R. and Hooker, D. C. 2018. Exploring Genotype $\times$ Environment $\times$ Management synergies to manage Fusarium head blight in wheat. Can. J. Plant Pathol. 40:179-188.

Berthiller, F., Dall'Asta, C., Schuhmacher, R., Lemmens, M., Adam, G. and Krska, R. 2005. Masked mycotoxins: determination of a deoxynivalenol glucoside in artificially and naturally contaminated wheat by liquid chromatography-tandem mass spectrometry. J. Agric. Food Chem. 53:3421-3425.

Boutigny, A.-L., Richard-Forget, F. and Barreau, C. 2008. Natural mechanisms for cereal resistance to the accumulation of $\mathrm{Fu}-$ sarium trichothecenes. Eur. J. Plant Pathol. 121:411-423.

Buerstmayr, H., Lemmens, M., Grausgruber, H. and Ruckenbauer, P. 1997. Breeding for scab resistance in wheat: inheritance of resistance and possibilities for in vitro selection. In: Fusarium head scab: global status and future prospects, eds. by H. J. Dubin, L. Gilchrist, J. Reeves and A. McNab, pp. 5258. CIMMYT, Mexico, Mexico, D.F.

Burt, C., Steed, A., Gosman, N., Lemmens, M., Bird, N., Ramirez-Gonzalez, R., Holdgate, S. and Nicholson, P. 2015. Mapping a Type 1 FHB resistance on chromosome 4AS of Triticum macha and deployment in combination with two Type 2 resistances. Theor. Appl. Genet. 128:1725-1738.

Bushnell, W. R., Hazen, B. H. and Pritsch, C. 2003. Histology and physiology of Fusarium head blight. In: Fusarium head blight of wheat and barley, eds. by K. J. Leonard and W. R. Bushnell, pp. 44-83. The American Phytopathological Society, St. Paul, MN, USA.

Desjardins, A. E. 2006. Fusarium mycotoxins: chemistry, genetics and biology. APS Press, St. Paul, MN, USA. 260 pp.

Diamond, H. and Cooke, B. M. 1999. Towards the development of a novel in vitro strategy for early screening of Fusarium ear blight resistance in adult winter wheat plants. Eur. J. Plant Pathol. 105:363-372.

Feekes, W. 1941. De tarwe en haar milieu. In: Verslagen van de Technische Tarwe Commissie, 17, ed. by W. Feekes, pp. 523888. Hoitsema, Groningen, The Netherlands.

Ganeshan, S. and Chibbar, R. N. 2017. A simple novel expedited spike culture-derived variation creation strategy in wheat. $\mathrm{Ce}$ real Res. Commun. 45:539-548.

Ganeshan, S., Drinkwater, J. M., Repellin, A. and Chibbar, R. N. 2010. Selected carbohydrate metabolism genes show coincident expression peaks in grains of in vitro-cultured immature spikes of wheat (Triticum aestivum L.). J. Agric. Food Chem. 58:4193-4201.

Ganeshan, S., Leis, M., Drinkwater, J. M., Madsen, L. T., Jain, J. C. and Chibbar, R. N. 2012. In vitro-cultured wheat spikes provide a simplified alternative for studies of cadmium uptake in developing grains. J. Sci. Food Agric. 92:1740-1747.

Gilbert, J. and Woods, S. M. 2006. Strategies and considerations for multi-location FHB screening nurseries. In: The Global Fusarium Initiative for International Collaboration: A Strategic Planning Workshop, eds. by T. Ban, J. M. Lewis and E. E. Phipps, pp. 93-102. International Maize and Wheat Improvement Center, El Batan, Mexico.

Huang, C., Gangola, M. P. and Chibbar, R. N. 2020. Utilization of wheat spike culture to assess Fusarium head blight disease progression and mycotoxin accumulation. Can. J. Plant Pathol. 42:62-71.

Huang, C., Gangola, M. P., Ganeshan, S., Hucl, P., Kutcher, H. R. and Chibbar, R. N. 2019. Spike culture derived wheat (Triticum aestivum L.) variants exhibit improved resistance to multiple chemotypes of Fusarium graminearum. PLoS ONE 14:e0226695.

Imathiu, S., Edwards, S., Ray, R. and Back, M. 2014. Artificial 
inoculum and inoculation techniques commonly used in the investigation of Fusarium head blight in cereals. Acta Phytopathol. Entomol. Hung. 49:129-139.

Jin, F., Zhang, D., Bockus, W., Baenziger, P. S., Carver, B. and Bai, G. 2013. Fusarium head blight resistance in U.S. winter wheat cultivars and elite breeding lines. Crop Sci. 53:20062013.

Kumar, K., Xi, K., Turkington, T., Tekauz, A., Helm, J. H. and Tewari, J. P. 2011. Evaluation of a detached leaf assay to measure Fusarium head blight resistance components in barley. Can. J. Plant Pathol. 33:364-374.

Kurata, H. and Ueno, Y. 1984. Toxigenic fungi: their Toxins and health hazard. In: Proceedings of the Mycotoxin Symposia Held in the Third International Mycological Congress, eds. by H. Kurata and Y. Ueno, pp. 1-363. Kodansha, Tokyo.

Lemmens, M., Scholz, U., Berthiller, F., Dall‘Asta, C., Koutnik, A., Schuhmacher, R., Adam, G., Buerstmayr, H., Mesterházy, A., Krska, R. and Ruckenbauer, P. 2005. The ability to detoxify the mycotoxin deoxynivalenol colocalizes with a major quantitative trait locus for Fusarium head blight resistance in wheat. Mol. Plant-Microbe Interact. 18:1318-1324.

Lulin, M., Yi, S., Aizhong, C., Zengjun, Q., Liping, X., Peidu, C., Dajun, L. and Xiu-e, W. 2009. Molecular cloning and characterization of an up-regulated UDP-glucosyltransferase gene induced by DON from Triticum aestivum L. cv. Wangshuibai. Mol. Biol. Rep. 37:785-795.

Marasas, W. F. O., Nelson, P. E. and Toussoun, T. A. 1984. Toxigenic Fusarium species, identity and mycotoxicology. Pennsylvania State University Press, University Park, PA. 328 pp.

McMullen, M., Bergstrom, G., De Wolf, E., Dill-Macky, R., Hershman, D., Shaner, G. and Van Sanford, D. 2012. A unified effort to fight an enemy of wheat and barley: Fusarium head blight. Plant Dis. 96:1712-1728.

McMullen, M., Jones, R. and Gallenberg, D. 1997. Scab of wheat and barley: a re-emerging disease of devastating impact. Plant Dis 81:1340-1348.

Mesterházy, A. 1987. Selection of head blight resistant wheats through improved seedling resistance. Plant Breed. 98:25-36.

Mesterházy, Á. 2002. Role of deoxynivalenol in aggressiveness of Fusarium graminearum and F. culmorum and in resistance to Fusarium head blight. Eur. J. Plant Pathol. 108:675-684.

Mesterházy, Á., Bartók, T., Mirocha, C. G. and Komoróczy, R. 1999. Nature of wheat resistance to Fusarium head blight and the role of deoxynivalenol for breeding. Plant Breed. 118:97110.

Miedaner, T., Sieber, A.-N., Desaint, H., Buerstmayr, H., Longin, C. F. H. and Würschum, T. 2017. The potential of genomicassisted breeding to improve Fusarium head blight resistance in winter durum wheat. Plant Breed. 136:610-619.

Miller, J. D., Young, J. C. and Sampson, D. R. 1985. Deoxynivalenol and Fusarium head blight resistance in spring cereals. $J$. Phytopathol. 113:359-367.
Parry, D. W., Jenkinson, P. and McLeod, L. 1995. Fusarium ear blight (scab) in small grain cereals: a review. Plant Pathol. 44:207-238.

Poppenberger, B., Berthiller, F., Lucyshyn, D., Sieberer, T., Schuhmacher, R., Krska, R., Kuchler, K., Glössl, J., Luschnig, C. and Adam, G. 2003. Detoxification of the Fusarium mycotoxin deoxynivalenol by a UDP-glucosyltransferase from Arabidopsis thaliana. J. Biol. Chem. 278:47905-47914.

Ruan, Y., Comeau, A., Langevin, F., Hucl, P., Clarke, J. M., Brule-Babel, A. and Pozniak, C. J. 2012. Identification of novel QTL for resistance to Fusarium head blight in a tetraploid wheat population. Genome 55:853-864.

Schroeder, H. W. and Christensen, J. J. 1963. Factors affecting resistance of wheat to scab caused by Gibberella zeae. Phytopathology 53:831-838.

Shah, L., Ali, A., Yahya, M., Zhu, Y., Wang, S., Si, H., Rahman, H. and Ma, C. 2018. Integrated control of Fusarium head blight and deoxynivalenol mycotoxin in wheat. Plant Pathol. 67:532-548.

Sharma, P., Gangola, M. P., Huang, C., Kutcher, H. R., Ganeshan, S. and Chibbar, R. N. 2018. Single nucleotide polymorphisms in B-genome specific UDP-glucosyl transferases associated with Fusarium head blight resistance and reduced deoxynivalenol accumulation in wheat grain. Phytopathology 108:124132.

Shin, S., Kim, K.-H., Kang, C.-S., Cho, K.-M., Park, C. S., Okagaki, R. and Park, J.-C. 2014. A simple method for the assessment of Fusarium head blight resistance in Korean wheat seedlings inoculated with Fusarium graminearum. Plant Pathol. J. 30:25-32.

Stack, R. W. and McMullen, M. P. 1995. A visual scale to estimate severity of Fusarium head blight in wheat. North Dakota State University, Fargo, ND, USA. 1095 pp.

Steiner, B., Buerstmayr, M., Michel, S., Schweiger, W., Lemmens, M. and Buerstmayr, H. 2017. Breeding strategies and advances in line selection for Fusarium head blight resistance in wheat. Trop. Plant Pathol. 42:165-174.

Ward, T. J., Clear, R. M., Rooney, A. P., O’Donnell, K., Gaba, D., Patrick, S., Starkey, D. E., Gilbert, J., Geiser, D. M. and Nowicki, T. W. 2008. An adaptive evolutionary shift in Fusarium head blight pathogen populations is driving the rapid spread of more toxigenic Fusarium graminearum in North America. Fungal Genet. Biol. 45:473-484.

Xue, A. G., Armstrong, K. C., Voldeng, H. D., Fedak, G. and Babcock, C. 2004. Comparative aggressiveness of isolates of Fusarium spp. causing head blight on wheat in Canada. Can. J. Plant Pathol. 26:81-88.

Yang, Z. P., Yang, X. Y. and Huang, D. C. 1999. Comparison of evaluation methods for selection of resistance to Fusarium head blight in a recurrent selection programme in wheat (Triticum aestivum L.). Plant Breed. 118:289-292. 